\title{
The MINOS long-baseline experiment at Fermilab *
}

\author{
David S. Ayres*
}

"High Energy Physics Division, Argonne National Laboratory, Argonne, Illinois 60439, U.S.A.

\section{RECEIVED SEP 211999 OSTI}

The MINOS long-baseline experiment will search for neutrino oscillations by measuring an intense $\nu_{\mu}$ beam at the end of a $730 \mathrm{~km}$ flight path. The 10,000 ton MINOS 'far detector' will utilize magnetired steel plates interleaved with track chambers to reconstruct event topologies and to measure the energies of the muons, hadrons and electromagnetic showers produced by neutrino interactions. The experiment is designed to detect $\nu_{\mu} \rightarrow \nu_{\tau}$ and $\nu_{\mu} \rightarrow \nu_{e}$ oscillations with $\Delta m^{2} \geq 0.001 \mathrm{eV}^{2}$ and $\sin ^{2}(2 \theta) \geq 0.01$. Any oscillation signal observed can be rerified and studied by several independent tests: a near/far rate comparison, the NC/CC event ratio, the CC and NC event energy spectra, and the identification of electrons and $T$ leptons. The neutrino beam can be operated in both wide-band and narrow-band configurations, allowing the detailed study oscillation phenomena. The experiment is scheduled to begin operation in 2001 .

\section{Introduction}

The MINOS (Main Injector Neutrino Oscillation Search) experiment will search for the oscillations of muon neutrinos into tau and/or electron neutrinos in an intense neutrino beam generated by Fermilab's new Main Injector accelerator. The experiment [1] is being mounted by the MINOS collaboration [2], which currently consists of 200 physicists and engineers from 22 institutions in the United States, the United Kingdom, Russia, and China.

The neutrino beam energy, $\left\langle E_{\nu}\right\rangle \approx 15 \mathrm{GeV}$, is above threshold for producing $\tau$ leptons, which will be important if $\nu_{\mu} \rightarrow \nu_{\tau}$ oscillations occur. The design of the neutrino beam will allow operation in both wide-band and narrow-band configurations, in order to verify and study any oscillation signal which is observed. The COSMOS short-baseline neutrino oscillation experiment [3] will use a hybrid emulsion detector in the same beam, just downstream of the $800 \mathrm{~m}$ decay tunnel on the Fermilab site.

Oseillations will be detected by comparison of neutrino event characteristics (e.g., rate, energy spectrum, NC/CC ratio) in a 'near' detector at Fermilab and a 10,000 ton 'far'. detector situated

"Submitted to the Proceedings of the 4th KEK Topical Conference on 'Flavor Physica,' KEK, Japan, 29-31 October 1986.
$730 \mathrm{~km}$ away in the Soudan underground laboratory. Measurements of neutrino events in the 1000-ton, very fine grained Soudan 2 tracking calorimeter, which is currently taking data on atmospheric neutrino interactions and searching for nucleon decay, will complement those in the much larger, coarser grained MINOS detector.

The beam trajectory is shown in Fig. 1. The $730 \mathrm{~km}$ distance gives an $L / E$ value which is well matched to the region of oscillation parameters, $\Delta m^{2}$ and $\sin ^{2}(2 \theta)$, suggested by the atmospheric neutrino flavor anomaly observed by the Kamiokande experiment [4]. The $\Delta m^{2}$ sensitivity depends on the ratio of beam length to energy, while the $\sin ^{2}(2 \theta)$ mixing angle sensitivity depends on the event rate (neutrino flux $\times$ cross section, and detector mass). For the wide-band beam configuration, the 10,000 ton far-detector mass gives an event rate of 20,000 charged current $\nu_{\mu}$ interactions per year in the absence of oscillations. This allows the detailed study of oscillations which occur with the Kamiokande parameters, $\Delta m^{2}=0.01 \mathrm{eV}^{2}$ and $\sin ^{2}(2 \theta)=0.7$. MINOS will also be able to explore a much larger region of oscillation parameter space, extending to quite low mixing angles, than that indicated by the atmospheric neutrino anomaly.

The sensitivity of an oscillation search can be limited by a number of systematic effects. One 


\section{DISCLAIMER}

This report was prepared as an account of work sponsored by an agency of the United States Government. Neither the United States Government nor any agency thereof, nor any of their employees, make any warranty, express or implied, or assumes any legal liability or responsibility for the accuracy, completeness, or usefulness of any information, apparatus, product, or process disclosed, or represents that its use would not infringe privately owned rights. Reference herein to any specific commercial product, process, or service by trade name, trademark, manufacturer, or otherwise does not necessarily constitute or imply its endorsement, recommendation, or favoring by the United States Government or any agency thereof. The views and opinions of authors expressed herein do not necessarily state or reflect those of the United States Government or any agency thereof. 


\section{DISCLAIMER}

Portions of this document may be illegible in electronic image products. Images are produced from the best available original document. 


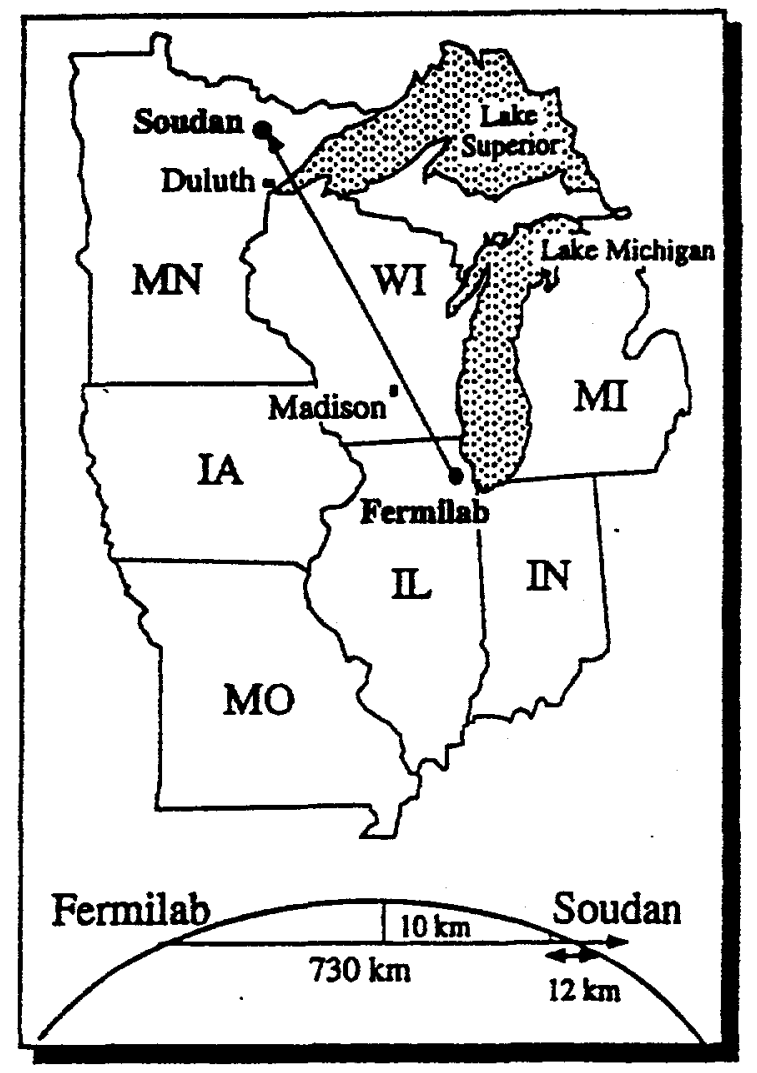

Figure 1. The trajectory of the MINOS neutrino beam between Fermilab and Soudan. The beam must be aimed into the earth at an angle of 57 mrad to reach Minnesota.

of the most important of these is the ability to predict the characteristics of the neutrino beam at the far detector in the absence of oscillations. For example, an observed difference from the expected event rate or energy spectrum at the far detector could be due to oscillations or to an incorrect extrapolation of beam characteristics from the near detector. Other systematic errors can arise from differences in near and far detector responses which are not properly accounted for. The latter effects will be minimized by making the near and far detectors as similar as possible.

\section{The MINOS far detector}

The initial feasibility studies for the experiment made use of a 'reference' detector design whose performance and construction cost could be predicted with some confidence. The 10,000 ton far reference detector consists of $6004-\mathrm{cm}$ thick magnetized iron planes interleaved with $32,000 \mathrm{~m}^{2}$ of limited streamer Iarocci tubes, read out by 480,000 electronics channels. The 8-m wide octagonal steel planes are toroidally magnetized with an average field of $1.5 \mathrm{~T}$. The $1-\mathrm{cm}$ square Iarocci tubes are read out by their anode wires (summed in pairs) and 2-cm wide external cathode strips. The far reference detector is shown schematically in Fig. 2. The estimated cost of the near and far reference detectors is $\$ 50 \mathrm{M}$; construction of the neutrino beam is expected to cost an additional \$50M.

Three different active detector technologies are now being considered for the MINOS far detector [5]: 'Iarocci' tubes (operated in either limitedstreamer or saturated-proportional mode) resistive plate counters (RPC's), and scintillator (either plastic or liquid) read out with wavelength shifting fibers. The detector design will be frozen in the fall of 1997 so that the first third of the far detector can be ready for beam turnon in the summer of 2001. Since the total detector construction cost is constrained to be $\$ 50 \mathrm{M}$ or less, the detector optimiration may involve 'trading off' mass (event rate) against performance, e.g., transverse and longitudinal (steel plane thickness) granularities.

\section{Design goals}

The MINOS detector design is optimized for the study of $\nu_{\mu} \rightarrow \nu_{\tau}$ oscillations with $\sin ^{2}(2 \theta) \geq$ 0.025 and $\Delta m^{2} \geq 0.001 \mathrm{eV}^{2}$. The $\sin ^{2}(2 \theta)$ sensitivity improves to 0.01 for $\nu_{\mu} \rightarrow \nu_{e}$ oscillations because of the higher $\nu_{e}$ cross section. If oscillations are observed, the experiment will determine the oscillation mode, or mixture of modes, and will measure the $\sin ^{2}(2 \theta)$ and $\Delta m^{2}$ parameters for each mode. The goal of the experiment is to achieve precisions of 0.1 in $\sin ^{2}(2 \theta)$ and $0.001 \mathrm{eV}^{2}$ in $\Delta m^{2}$. The mixture of $\nu_{\mu} \rightarrow \nu_{e}$ and $\nu_{\mu} \rightarrow \nu_{\tau}$ 


\section{MINOS (Main Injector Neutrino Oscillation Search)}

\section{Far Detector}

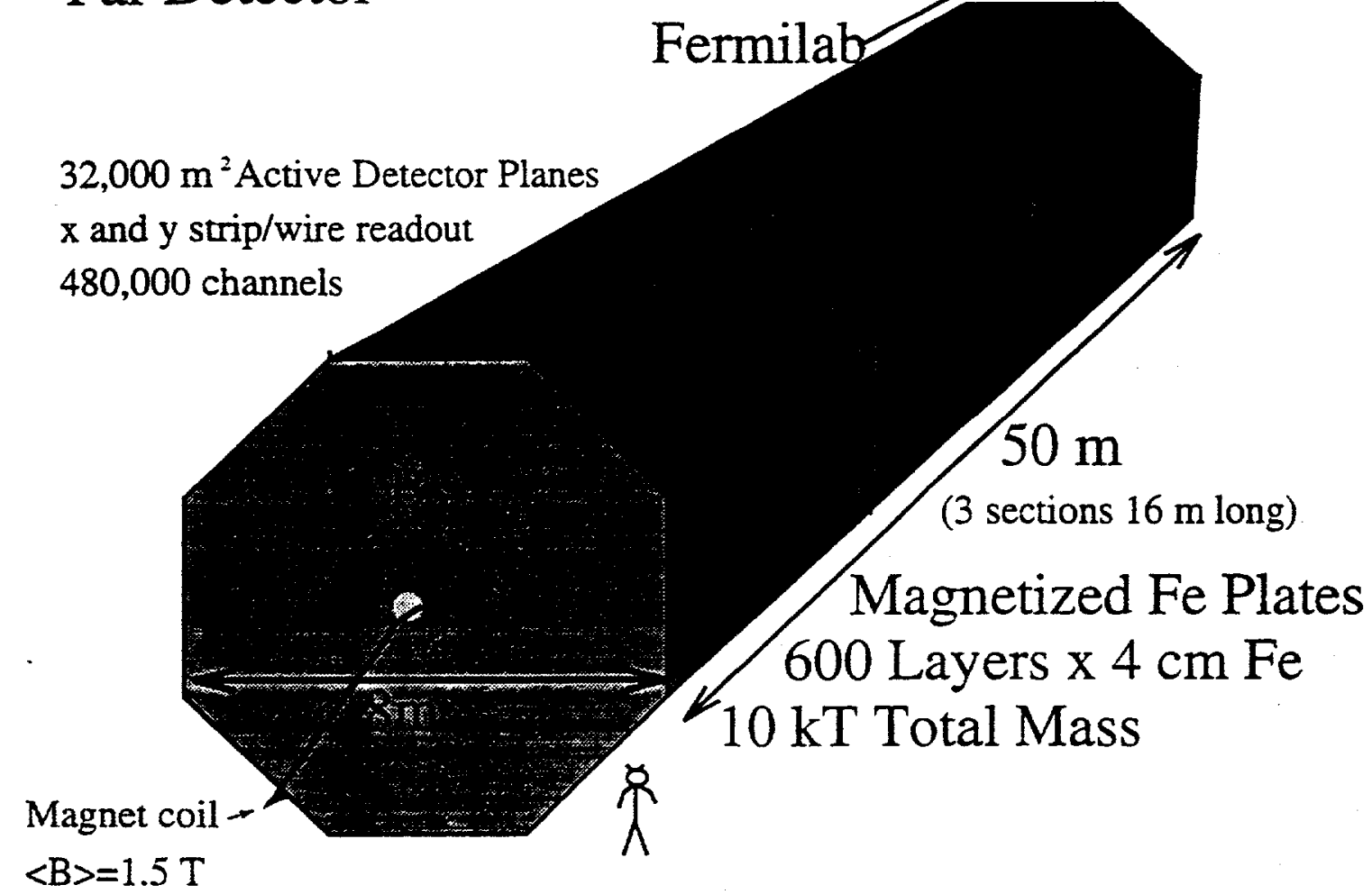

Figure 2. The MINOS reference detector, located $700 \mathrm{~m}$ underground in Soudan, Minnesota.

will be measured with an accuracy in $\sin ^{2}(2 \theta)$ of $\sim 0.05$. If $\nu_{\mu} \rightarrow \nu_{\tau}$ occurs with $\Delta m^{2} \sim 0.01 \mathrm{eV}^{2}$, the experiment should be able to identify exclusive $r$ lepton decay modes for $\sin ^{2}(2 \theta) \geq 0.4$.

\section{Oscillation tests}

Any oscillation signal observed can be verified and studied using two or more independent oscillation tests. These involve comparisons between the near and far detectors of quantities such as the neutrino event rate, the NC/CC ratio, the energy spectra of CC and NC events, electron and $\tau$ lepton appearance. Differences in the beam characteristics and detector responses at the two locations must be properly accounted for. A real oscillation signal will involve a self consistent pattern of effects in several tests. An oscillation signal can be further verified and studied by using the narrow-band beam to measure its energy dependence.

The projected sensitivities of several MINOS oscillation tests are compared to those of previous experiments in Fig. 3.

\subsection{Event topology classification}

Charged current (CC) $\nu_{\mu}$ interaction events recorded in the MINOS near and far detectors will usually contain long muon tracks and can be cleanly selected by a simple event length cut. If there are no oscillations, short-length events will be mainly neutral current (NC) $\nu_{\mu}$ interactions, 


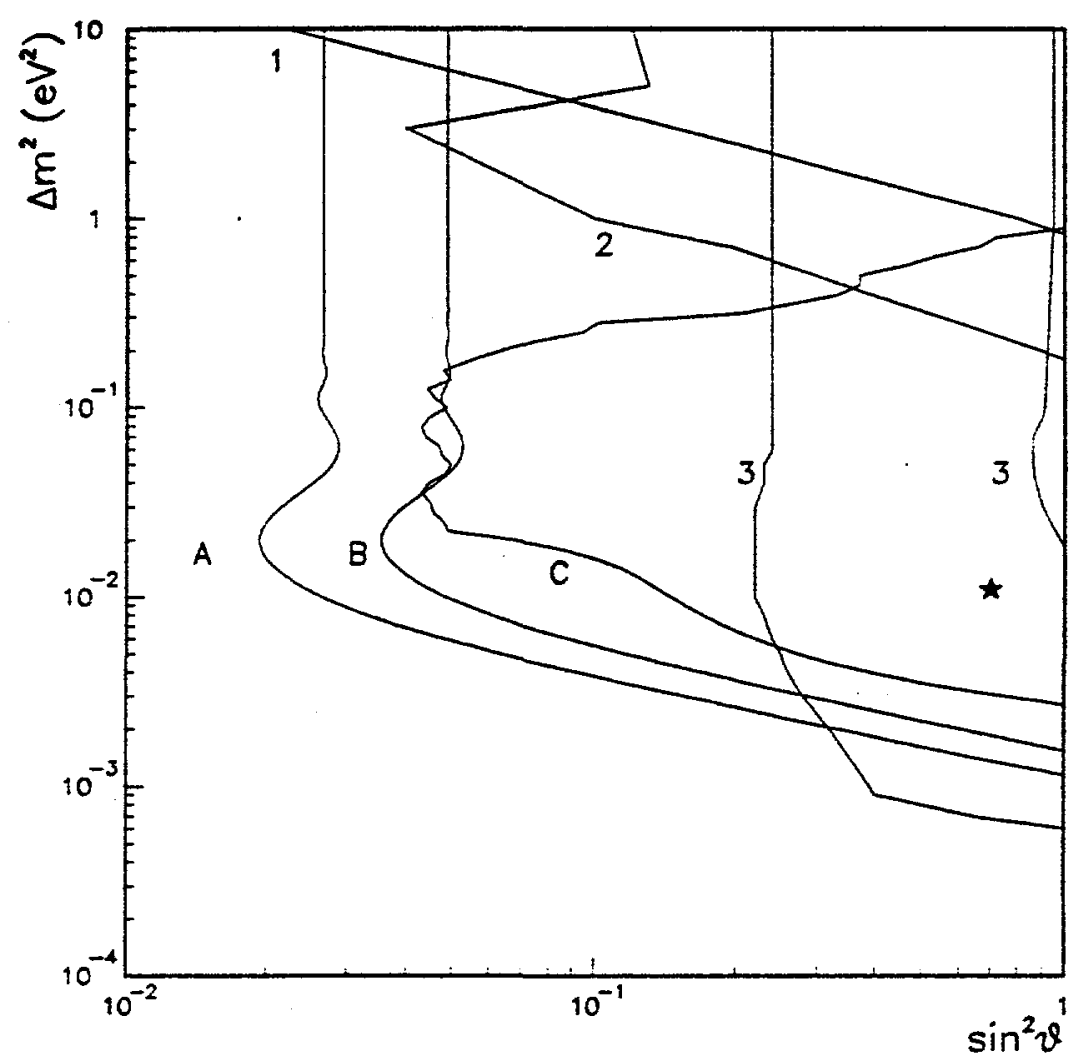

Figure 3. Comparison of $90 \%$ confidence level limits on $\nu_{\mu} \rightarrow \nu_{\tau}$ oscillation parameters from past experiments with those which will be obtained by MINOS in the absence of oscillations. Curves $A$, $B$, and $C$ are for the MINOS NC/CC test, the near/far test, and the CC event energy test respectively. Curves 1 and 2 are existing limits from Fermilab E-531 and CDHS respectively. The star symbol shows the Kamiokande atmospheric neutrino best fit parameters, and the two curves labeled 3 show the boundaries of the allowed region from that experiment.

but the NC sample will also include $\nu_{e}$ and $\nu_{\tau}$ charged current events if oscillations occur. The ability to identify CC interactions caused by electron or tau neutrinos, in the presence of a large background of muon neutrino events, will be a very important tool in the search for neutrino oscillations.

\subsection{Comparison of neutrino event rates}

The measurement of the relative rates of $\nu_{\mu} \mathrm{CC}$ events in the near and far detectors is the simplest form of disappearance experiment. However, several other effects can also cause significant nearfar rate differences, and corrections for these must be calculated. The most serious of these is the dif- ference between the neutrino beam energy spectra at the two locations. The root cause of these differences is the fact that the near detector views the $800 \mathrm{~m}$ long neutrino source (the decay tunnel) from only $500 \mathrm{~m}$ away, whereas the source appears as a point to the far detector. Corrections for this (energy dependent) effect will be $\leq 5 \%$ and require a precise model of the beam which includes neutrinos produced by beam scraping and an allowance for errors in alignment. Corrections are also required for differences in detector efficiencies and acceptances - the relative beam and detector sizes are very different at the two locations. The near/far test sensitivity shown as Curve B 
in Fig. 3 is dominated by an estimated total systematic error of $2 \%$.

\section{3. $\mathrm{NC} / \mathrm{CC}$ event ratio}

The comparison of the NC/CC ratios measured at the near and far detectors is the oscillation test which the collaboration has studied in the greatest detail. This is believed to be the most sensitive MINOS test because the effects of many differences between the near and far detectors and beam characteristics will cancel to first order. The test is performed by counting the numbers of ' $\mathrm{CC}$ ' and ' $\mathrm{NC}$ ' events in each detector and calculating the quantity $T=C C /(N C+C C)$. The measured value of $\mathrm{T}$ at the near detector is corrected for beam and detector differences (using simulations which are tuned to reproduce near detector measurements) to obtain the value of $T$ which is expected at the far detector in the absence of oscillations. A two year run with the full 10,000 ton MINOS far detector in the wide-band beam would yield a statistical error $\delta T=0.0020$, to be compared with an estimated systematic error of $\delta T=0.0008$. This is the most sensitive MINOS test, and is shown as Curve $A$ in Fig. 3.

\subsection{CC and NC event energy spectra}

Because the oscillation probability depends on neutrino energy, a real oscillation signal will include characteristic changes in the event energy spectra [6]. The measurement of event energy is therefore central to the study of any oscillation signal observed; the following resolutions for muons, hadrons, and electromagnetic showers are expected for the MINOS detectors:

$(\Delta p / p)_{\mu} \approx 10 \%$ (range and/or curvature), $(\Delta E / E)_{\text {hadron }} \leq 100 \% / \sqrt{E}$, and $(\Delta E / E)_{\text {em }} \leq 40 \% / \sqrt{E}$.

Figure 4 shows a Monte Carlo simulation of the CC event energy spectrum which would be observed by MINOS during the first calendar year of data taking (with one third of the far detector in operation) with the wide-band neutrino beam, assuming the Kamiokande best fit $\nu_{\mu} \rightarrow \nu_{\tau}$ OBcillation parameters. If such an oscillation signal were to be confirmed by other tests, it would be studied in detail using the narrow-band beam configuration. Although this beam provides (at the best energy) only about one fifth of the neutrino flux of the wide-band beam, its narrow energy spread allows a more accurate measurement of $\Delta \mathrm{m}^{2}$ as well as the determination of oscillation modes. The sensitivity of the CC-event energy test, shown as Curve $\mathrm{C}$ in Fig. 3, falls off for large values of $\Delta m^{2}$ where oscillations are rapid and bave the effect of lowering the overall rate of $\nu_{\mu} \mathrm{CC}$ events without changing the shape of the energy spectrum.

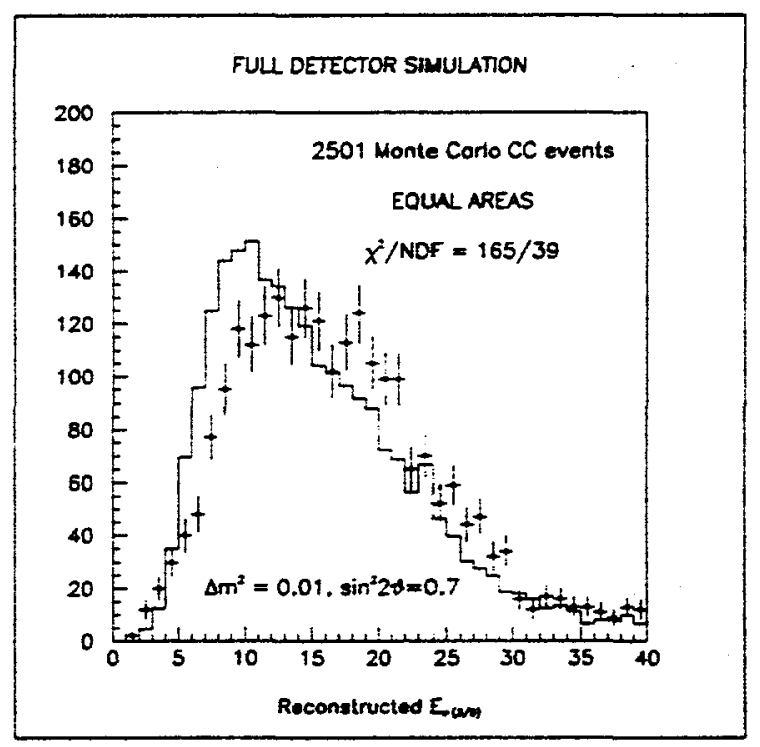

Figure 4. Expected wide-band-beam CC-event energy spectra for $\nu_{\mu} \rightarrow \nu_{\tau}$ oscillations (points with error bars) with $\Delta m^{2}=0.01 \mathrm{eV}^{2}$ and $\sin ^{2}(2 \theta)=0.7$, and for the no oscillations (solid histogram). The $\chi^{2} / \mathrm{df}=165 / 39$ value assumes that the absolute normalization is completely unknown, i.e., that only the shapes are compared. The oscillation data correspond to the exposure expected during the first calendar year of data ( 9 months of live time) with one third of the far detector in operation.

Figure 5 shows the differences, for three oscillation modes, between far detector energy spec- 


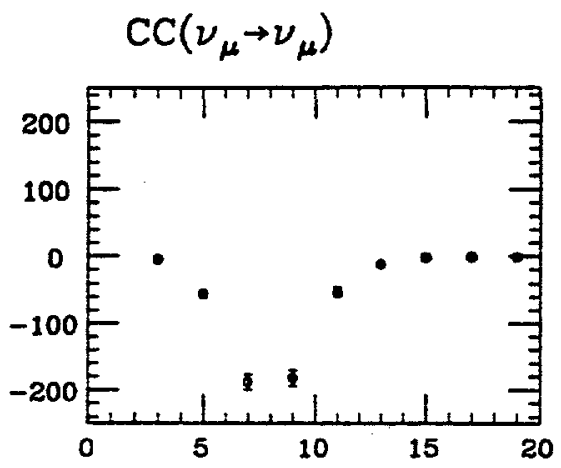

(A) Etotal(GeV)

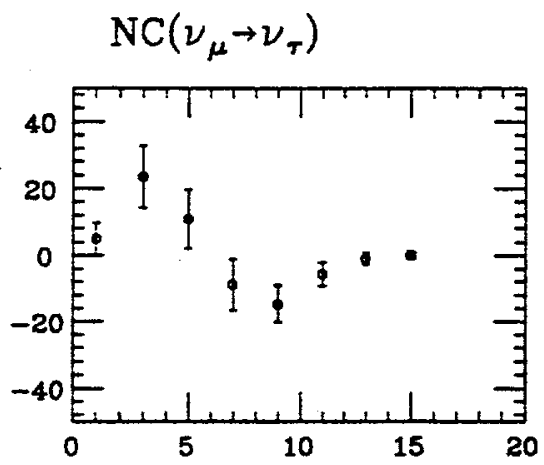

(C) $\operatorname{Ehad}(\mathrm{GeV})$

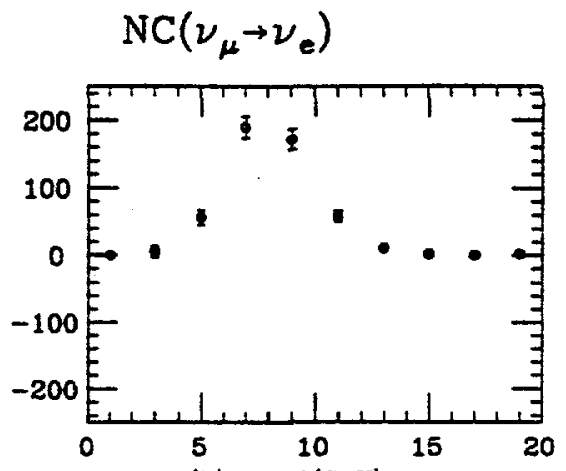

(B) Ehad(GeV)

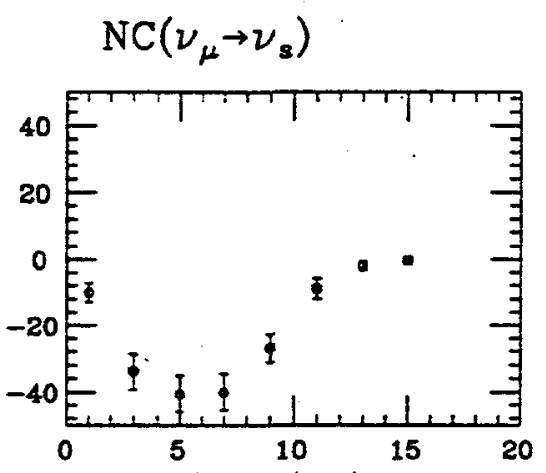

(D) $\operatorname{Ehad}(\mathrm{GeV})$

Figure 5. Expected energy spectrum differences for oscillations with $\Delta m^{2}=0.01 \mathrm{eV}^{2}$ and $\sin ^{2}(2 \theta)=0.7$, for three different oscillation modes. The simulated data correspond to a one-year exposure with the 8.4 GeV narrow-band beam. The quantities plotted are the differences between the data with oscillations and the predicted no-oscillation spectra.

tra with no oscillations and with the Kamiokande oscillation parameters. The simulated data correspond to 1 year of operation ( 1000 events) with the narrow-band beam tuned for $\left\langle E_{\nu}\right\rangle=$ $8.4 \mathrm{GeV}(20 \mathrm{GeV}$ pion energy). Differences in $\mathrm{CC}$ and 'NC' energy spectra are shown for three different oscillation hypotheses. Oscillations of the dominant $\nu_{\mu}$ component result in the depletion of CC events at the far detector. If the oscillations are $\nu_{\mu} \rightarrow \nu_{e}$, those events will show up as NC-like events with the full beam energy. If the oscillations are $\nu_{\mu} \rightarrow \nu_{\tau}$, a fraction of the lost CC events will show up as NC-like events but with lower visible energy. For $\nu_{\mu} \rightarrow \nu_{\text {sterile, }}$, there will be a decrease in the number of NC-like events which is proportional to that in the CC events.

\subsection{Electron and $\tau$ identification}

The detection of individual $\nu_{\varepsilon}$ or $\nu_{\tau} \mathrm{CC}$ interactions in the MINOS far detector, above the rates expected from background processes and beam contamination, would be clear evidence for oscillations. Quasi-elastic $\nu_{e}$ interactions at low $y$ will appear as unaccompanied electromagnetic showers with the full beam energy (modulated by the energy dependent oscillation probability) and 
should be easy to identify even with the wideband beam and the $4 \mathrm{~cm}$ thick steel planes of the reference detector. The beam contamination of $0.7 \% \nu_{\varepsilon}+\bar{\nu}_{e}$ from muon and kaon decays will be well measured by the near detector and will provide a useful calibration of detector response.

The identification of $\tau$ leptons produced in $\nu_{\tau}$ charged current interactions will be much more difficult. The number of $\nu_{T}$ interactions in a given decay mode is small because of the small branching fractions (18\% each for $\tau \rightarrow e \nu \nu$ and $\tau \rightarrow \mu \nu \nu$ ) and because (for example, with the Kamiokande oscillation parameters and the wideband beam) only a small fraction of the beam neutrinos are $\nu_{\tau}$ 's $(36 \%)$, the ratio of $\nu_{\tau}$ to $\nu_{\mu}$ CC cross sections is low (0.242) at these energies, and the hard cuts required to reduce the background from $\nu_{\mu}$ interactions by a factor of $\sim 100$ are likely to have a low acceptance $(\sim 10 \%)$ for $\tau$ decays.

The observation of explicit signatures of $\nu_{\tau}$ CC interactions in MINOS will rely on statistical methods (i.e., including background subtractions) based on kinematic properties of the final state. Although there are fewer quasi-elastic than deep-inelastic $\nu_{\tau}$ interactions, their simpler topologies and ease of reconstruction make them the best candidates for the identification of explicit $T$ appearance signatures. The relevant kinematic properties of $\nu_{\tau} \mathrm{CC}$ events are missing transverse momentum, using either the wideband or narrow-band beams, and missing total momentum using the narrow-band beam (due to the fact that $\tau$ decays produce neutrinos).

Although the energy constraint provided by the narrow-band beam will improve background rejection, event rates will be about a factor of five lower in the narrow band beam than in the wide band beam. Furthermore, systematic uncertainties can be as important as statistical limitations. For example, the low-energy 'tail' of the narrow-band beam energy spectrum and the nonGaussian nature of detector resolution functions can produce large backgrounds. Much MINOS analysis effort is now being devoted to improving the understanding of these two crucial issues.

The identification of $\tau \rightarrow \pi X$ decays, which have a relatively high branching fraction ( $12 \%$ for $\tau \rightarrow \pi \nu$ ), appears to be feasible using the signature of a single, high-energy charged pion. High energy pions are more likely to be produced in $T$ decays than in NC interactions. Distinctive event topologies consisting of a single long track ending in a multiprong interaction will be relatively easy to identify, particularly if the thickness of MINOS steel planes can be reduced from $4 \mathrm{~cm}$ to $2 \mathrm{~cm}$. MINOS simulations indicate that the identification of $\tau \rightarrow \pi X$ events may be possible in the wide-band beam, where the main background comes from neutral current interactions of the high-energy tail of the beam.

Our preliminary analyses suggest that MINOS should be able to observe $\nu_{\mu} \rightarrow \nu_{\tau}$ oscillations with the Kamiokande parameters with a statistical significance greater than $3 \sigma$ in a variety of decay modes, using both the narrow-band and wide-band beams. This corresponds to $90 \%$ confidence level limits on $\sin ^{2}(2 \theta)$ between 0.2 and 0.3 .

Finally, the collaboration has recently begun to consider an informal proposal made by $\mathrm{K}$. Niwa of the Nagoya University group. Tau decays would be identified in a hybrid emulsion detector by selecting events with a secondary vertex impact parameter or a decay 'kink.' The 1000 ton modular detector would be constructed of alternating layers of 1-mm thick steel and emulsion-coated plastic. The $\sim 50 \mu \mathrm{m}$ thick layers of emulsion (manufactured by X-ray film production technology) would be laminated to both sides of $1-\mathrm{mm}$ thick plastic sheets, and would be relatively inexpensive. Candidate $\tau$ decay events would be located by gas tracking chambers to limit the amount of emulsion to be measured. Very preliminary simulations show that such a detector, installed at Soudan, could cleanly identify a useful number of $\tau$ decays in a few years of operation. It might even be possible to remove and measure emulsion modules as candidate events occur, instead of waiting until the exposure was completed. Discussions between MINOS and Nagoya are still in very preliminary stages and detailed simulation work is just getting under way. 


\section{Outlook}

The MINOS experiment is designed to detect and study neutrino oscillations in a large region of parameter space around the $\Delta m^{2}$ and $\sin ^{2}(2 \theta)$ values suggested by the atmospheric neutrino flavor ratio anomaly. If oscillations do not occur, the experiment will set new, quite restrictive limits. If oscillations do occur however, the MINOS detectors and neutrino beam are designed with the sensitivity and flexibility to be able to characterize the phenomenon in considerable detail. Under favorable conditions the experiment will determine the mix of oscillation modes and measure oscillation parameters.

As described at this conference by J. Sato [7], neutrino oscillation experiments can test CP conservation by searching for a difference between the oscillation probabilities for $\nu_{\mu} \rightarrow \nu_{e}$ and $\bar{\nu}_{\mu} \rightarrow \bar{\nu}_{e}$, which are required to be equal by $C P$ symmetry. Such a test could be compromised by matter effects if $\Delta m^{2}$ were very small $\left(\sim 10^{-5} \mathrm{eV}^{2}\right)$, but should in principle be possible for MINOS [8] if the oscillation parameters are favorable and suffcient $\bar{\nu}_{\mu}$ beam intensity could be obtained. Matter effects are even less important for the CPviolation test using $\nu_{\mu} \rightarrow \nu_{\tau}$ and $\bar{\nu}_{\mu} \rightarrow \bar{\nu}_{\tau}$, which does not involve electron neutrinos directly (the $\nu_{\tau}$ and $\nu_{e}$ modes are related by unitarity).

The MUNOS collaboration is currently developing a very detailed Monte Carlo simulation of the experiment, including realistic descriptions of the neutriso beam properties, neutrino interactions, and detector response. This will allow us to evaluate the sensitivity of the various oscillation tests using different detector and beam parameters, for example transverse and longitudinal granularities, scintillator us gas active detector technologies, and neutrino beam energy spectra. The designs will be frozen during the summer of 1997 so that the beam and detector will be ready to operate in 2001.

I would like to thank M. Goodman and S. Pakvasa for enlightening discussions of the material presented here, and R. Seidlein for help with the the preparation of this manuscript. MINOS design and development work is supported by the U.S. Department of Energy, the U.S. National
Science Foundation and the U.K. Particle Physics and Astronomy Research Council. Substantial support has also been provided by the individual universities, laboratories and research institutions of the MINOS collaboration.

\section{REFERENCES}

1. The MINOS Collaboration, "P-875: A longbaseline neutrino oscillation experiment at Fermilab," NuMI-L-63, Feb. 1995.

2. The following institutions are currently members of the MINOS Collaboration:

Argonne, Caltech, Columbia, Fermilab, IHEP-Beijing, IHEP-Protvino, Indiana, ITEP-Moscow, JINR-Dubna, Lebedev, Livermore, Minnesota, Oak Ridge, Oxford, PNPISt.Petersburg, Rutherford, Stanford, Sussex, Texas A\&M, Texas Austin, Tufts, Western Washington.

3. N.W. Reay (spokesman) et al., "E-803 pro posal for a short baseline experiment at Fermilab," October 1990; revised October 1993.

4. Y. Fukuda, et al., Phys. Lett. B335 (1994) 237.

5. D.S. Ayres, "Detectors for the MINOS longbaseline neutrino oscillation experiment," to be published in the Proceedings of the 5th Int. Conf. on Advanced Technology and Particle Physics, Como, Italy, 7-11 October, 1996 (Nuclear Physics B Proceedings Supplement).

6. J.H. Cobb, Nucl. Instr. and Methods A373 (1996) 501.

7. J. Sato, " $\mathrm{CP}$ and $\mathrm{T}$ violation test in neutrino oscillation," talk presented at this meeting; also, J. Arafune and J. Sato, preprint hep$\mathrm{ph} / 9607436$, to be published in Phys. Rev. D.

8. S. Pakvasa, private communication, and $M$. Tanimoto, preprint hep-ph/9605413. 